\title{
Effect of taping as a component of conservative treatment for subacromial impingement syndrome
}

\author{
Natesagounder Sundaramurthy Senthil Kumar ${ }^{1^{*}}$, Arun Nehru ${ }^{2}$, Dharmarajan Rajalakshmi ${ }^{1}$ \\ ${ }^{1}$ Faculty of Therapeutic Sciences, Masterskill University College of Health Sciences, Cheras, Malaysia; \\ *Corresponding Author: nsshakthi@gmail.com \\ ${ }^{2}$ Christian Medical College and Hospital, Vellore, India
}

Received 7 December 2011; revised 11 January 2012; accepted 2 February 2012

\section{ABSTRACT}

Subacromial impingement syndrome (SIS) has been described as the most common form of shoulder pathology argued that $100 \%$ of impingement lesions and $95 \%$ of rotator cuff pathology are caused by friction between the acromion and surrounding tissues within the subacromial space. Commonly, rest, Non-steroidal anti-inflammatory drugs (NSAIDS), corticosteroid injections, and mobilization, strengthening exercises will resolve most cases of SIS. The results of the long-term outcome of these treatments, however, are not promising. Purpose: This study focuses on the effect of scapular taping and conventional Physical therapy in increasing isometric muscle strength, decreasing pain, improving function in patients with subacromial impingement syndrome. Study design: Double blinded randomised controlled clinical trial. Subjects were assessed by a blinded researcher using standard physiotherapy measures, Shoulder Pain and Disability Index questionnaire (SPADI), and isometric muscle strength measured using digital dynamo meter. Methodology: The patients in the treatment group (scapular taping group) received scapular taping with conventional exercises and the control group (no scapula taping) group received conventional exercise. Result: When comparing the scores using unpaired t-test with $p$-value set at $<0.05$, high level of significance was noted for flexor, abductor, external rotator muscle force and SPADI. Conclusion: Scapular taping may be a useful adjunct for promoting proper scapular kinetics \& upper thoracic extension and should be used in conjunction with other interventions.

Keywords: Shoulder Impingement Syndrome; Impingement; Scapular Taping; Exercise

\section{INTRODUCTION}

Shoulder disorders are among the most common of all peripheral joint complaints. The cumulative incidence of shoulder problems is estimated to be 11.2/1000 patients per year [1]. SIS and rotator cuff tendinitis are considered to be the most frequent cause of intrinsic shoulder pain and disability. SIS is a condition where sub acromial soft tissue structures compressed against or under anterior acromion by head of humerus on overhead activities and thus manifest as anterior or anterior-lateral shoulder pain. The pain is associated with a loss of shoulder function, especially during overhead activities, occurring during vocational, sporting or the normal activities associated with daily living. Pain and dysfunction occur when the shoulder is placed in positions of elevation, an activity that is common place during many sporting and vocational pursuits, and activities involved in daily living $[2,3]$. SIS has been associated with shoulder pain in a number of sports, including golf, volleyball, badminton, basketball, tennis, cricket, swimming and baseball. Ischemia, inflammation, and degeneration are related to age and overload of the tendons of the short rotator muscles and are present in rotator cuff disease [4,5]. The etiology of sub acromial impingement is multifactorial; these causes include anatomical and mechanical factors, rotator cuff pathology, glenohumeral instability, restrictive processes of the glenohumeral joint, imbalance of the muscles controlling the scapula, and postural considerations. Neer (1972) stated that impingement occurred mainly when the arm was in a position of forward elevation. He labeled the site of compression as the impingement zone, and hypothesized that spurs located at the acromial end of the coracoacromial ligament led to wear and tear to the rotator cuff. He proposed that SIS occurred in three stages and this could be viewed as a pathological process in continuum: acute inflammation (tendinitis or bursitis) (stage I); degeneration or chronic inflammation, or both (stage II); rupture and arthritis (stage III).Commonly, rest, analgesics, anti-inflammatory drugs, 
local steroid injections, and mobilization with simple exercises will resolve most cases of tendinitis. The results of the long term outcome of these treatments, however, are not promising.

Studies have reported a 95\% "success" rate for returning an athlete to his or her prior level of competition within 3 months without surgery [6]. Unfortunately, this report contained no data to support on scapular taping. Hence this study focuses on the effect of taping, and conventional therapy in increasing isometric muscle strength, decreasing pain, improving function in a patient with subacromial impingement syndrome.

\section{MATERIALS AND METHODS}

The purpose of this study is to demonstrate the use of scapular taping in patients with shoulder impingement syndrome.

\subsection{Study Design}

Double blinded randomised controlled clinical trial was used. Subjects were randomly assigned to either the experimental (scapular taping) or the control (no scapular taping) group. Both the subject and assessor were blinded to group assignment. Treatment was administered by the principal investigator.

\subsection{Subjects}

Thirty six male and sixteen female patients (mean age -36.2 yr) diagnosed by their referring physicians with SIS were enrolled. Subject selection criteria includes unilateral shoulder pain of more than 1 week localized (anterior and/or anterolateral) to the acromion and pain produced or increased during flexion and/or abduction of the symptomatic shoulder and at least any four of the following [7]: Positive Neer impingement sign, Positive Hawkins sign, Pain reproduced during supraspinatus empty can test, Painful arc of movement between $60^{\circ}$ to $120^{\circ}$, Pain with palpation on the greater tuberosity of the humerus.

Differential examination performed by the principal investigator, aided by information provided by the referring physician, ruled out cervical radiculopathy or radiculitis, a rotator cuff tear, adhesive capsulitis, history of shoulder dislocation, subluxation or fracture, history of cervical, shoulder, or upper back surgery, shoulder instability, calcifying tendonitis, degenerative arthritis of the glenohumeral joint \& acromioclavicular joint. In some cases, clinical tests were supplemented with information from physician-interpreted radiographic, and computerized tomography scan studies. All participants were then assessed by a blinded researcher using standard physiotherapy measures and they completed a Shoulder Pain and Disability Index questionnaire (SPADI), and isometric muscle strength measured using digital dynamometer.

\subsection{Group I Experimental Group (Scapular Taping Group)}

The patients in this group received scapular taping along with conventional therapy. All the patients received treatment for three days a week for 6 weeks. Taping is based on the presence of a posture impingement relationship. People with increased thoracic kyphosis and forward head posture have increased risk of developing SIS. The treatment technique that was used was that advocated by Lewis et al. Real postural change was achieved by requesting the subjects to extend their thoracic spines. The investigator demonstrated the movement to each subject and the subjects were allowed to practice this once before the application of 3.8-cm-wide Leukotape which was applied bilaterally from T1 to T12 [8].

Subjects were then asked to fully retract and depress their scapula, and tape was applied from the centre of the spine of the scapula to the T12 spinous process in a diagonal fashion. The leukotape was tensed prior to application on the subject and the subjects maintained the required postural changes while the tape was applied. Subjects were not required to actively maintain the postural change, as the aim of the tape was to hold each subject in the new posture. Patients must return to the outpatient clinic thrice a week to be re-taped, as they cannot tape themselves. On the first day of treatment the patients were taught conventional exercises and each of which was reviewed during following sittings.

The tape should never restrict a patient's range of motion. Additionally, it should allow the patient to perform motions that would have been painful without the tape in place, thus providing an immediate assessment of the tape's effectiveness.

\subsection{Group II Control Group (Conventional Treatment)}

The patients in this group were treated with conventional exercises consist of icing, joint mobilisation, stretching and strengthening exercises. All subjects received cold packs, active range of motion, physiologic stretching and muscle strengthening exercises, soft tissue mobilization, and patient education. In the supine position, cold packs were administered for 15 minutes with placement covering the posterior, superior, and anterior aspects of the shoulder. Active range of motion exercises consisted of pendulum exercise and postural correction performed within pain-free range. Physiologic stretching consisted of cane-assisted flexion and external rotation, towel-assisted internal rotation, and noninvolved arm- 
assisted horizontal adduction, performed within tolerable limits, preferably to the end of available range. Muscle strengthening exercises included chair press and internal and external rotation isometrics. The postural correction and chair press exercise focused on correction of postural imbalance, including scapular asymmetry. All other exercises were designed to restore synchronous scapulohumeral rhythm either through stretching to restore glenohumeral capsular mobility or strengthening to restore strength and timing of the rotator cuff and parascapular musculature [9]. Subjects were instructed to avoid increased pain with all exercises and daily activities and were advised to position the upper extremity in a supported $40^{\circ}-50^{\circ}$ scapular plane elevation position (loose packed position) when not using the extremity [10]. This positioning not only helps to maximize the precarious vascularity of the cuff but also helps to minimize excessive tightening of the inferior capsule [11]. Each subject's daily exercises and vocational aid avocational activities were reviewed to ensure good quality exercise and avoidance of improper mechanics or overuse stress [12].

\subsection{Outcome Measures}

Measurement were taken prior to randomization at baseline and then at 6 weeks following the commencement of treatment. All data were collected by an assessor blinded to the patient's group allocation.

The primary outcome measure used for the study was the SPADI questionnaire and isometric muscle strength. SPADI is a self-administered questionnaire yields a continuous measure between 0 to 100 on 13 items measuring shoulder pain and functional status. These are used to produce two subscale scores for pain (5 items) and disability (8 items), and a total score. This instrument has previously been demonstrated to be valid and reliable in detecting changes in patient clinical status [13-15].

Shoulder isometric strength was evaluated using a digital dynamometer using the standardized approach described and assessed for both inter and intra-rater reliability by Brain et al. The Isobex 2.0 fixed dynamometer is a compact, light weight and portable device. It requires fixation to a smooth surface by two suction pads. Force exerted is measured through a steel wire elongated with an adjustable strap for fixation to the patient. Force is displayed in kilograms followed by a three second isometric contraction. The subjects exerted a maximum isometric muscle contraction lasting 3 seconds while the dynamometer was held stationary. This was repeated 3 times with a $30 \mathrm{sec}$ rest period, with the examiner issued instructions and encouragement to promote maximal effort in each test positions. Two-minute rest periods were given between contraction measurements of different muscle groups [16].

\section{DATA ANALYSIS}

Three patients who could not be present for the duration of the study were excluded from data analysis. Measurements of all outcomes were examined for normality and taping and control groups using measurements from baseline and 6 weeks following the commencement of treatment. A p-value of less than 0.05 was considered to be statistically significant.

Table 1 Comparing with the unpaired t-test, the scapular taping group was improved by 6.30 units when compared to the control group. It was concluded that scapular taping is more effective in enhancing shoulder function that conventional therapy.

Table 2 show that the isometric muscle strength (in kg) in the taping group has increased than the control group and the highly significance difference noted in the flexors, abductors and external rotators and significant difference was noted in the extensors and internal rotators measures. Thus taping with conventional exercise is proven to be effective in increasing muscle strength when compared to conventional exercise alone.

\section{RESULTS}

At the baseline of the study, there were no differences between groups on the variables of SPADI pain and disability and the isometric strength. Following treatment, the taping group demonstrated improved score in SPADI demonstrating a decrease in pain and disability and improvement in isometric muscle strength compared with the control group.

\section{LIMITATION}

There is the possibility the "hands-on" treatment of scapular taping therapy is perceived by the patient as more intensive care compared to no taping treatment. Further the study lacked long-term follow up and this could be included in future studies.

\section{DISCUSSION}

There are several factors which will determine the success of a patient treatment with SIS. Firstly, improving the biomechanics of the scapulohumeral and scapulothoracic joints is one that ultimately relieves the patient's symptoms. Scapular taping may be one way to improve scapular alignment. Holding the scapula in better alignment with tape may provide a prolonged stretch to the tight structures around the shoulder. Additionally, this improvement in position helps to increase the subacromial space. Thus, the taping may relieve any excessive tension placed on the involved structures of the impinge- 
Table 1. Spadi score.

\begin{tabular}{cccccc}
\hline GROUP & Mean & SD & SEM & p-value & t-value \\
\hline Taping & 52.23 & 9.79 & 1.92 & & \\
Control & 45.92 & 9.51 & 1.86 & 0.024 & 2.36 \\
Diff & \multicolumn{2}{c}{6.31} & 2.67 & & \\
\hline
\end{tabular}

Table 2. Isometric muscle strength.

\begin{tabular}{|c|c|c|c|c|c|}
\hline Movement & GROUP & Mean & SD & SEM & $\mathrm{t}$-value \\
\hline \multirow{3}{*}{ Flexion } & Taping & 1.22 & 0.45 & 0.08 & \\
\hline & Control & 0.56 & 0.20 & 0.04 & 5.32 \\
\hline & Diff & \multicolumn{2}{|c|}{0.66} & & \\
\hline \multirow{3}{*}{ Extension } & Taping & 0.96 & 0.65 & 0.12 & \\
\hline & Control & 0.56 & 0.22 & 0.04 & 2.97 \\
\hline & Diff & \multicolumn{2}{|c|}{0.40} & & \\
\hline \multirow{3}{*}{ Abduction } & Taping & 1.66 & 0.46 & 0.09 & 4.30 \\
\hline & Control & 1.14 & 0.40 & 0.08 & \\
\hline & Diff & \multicolumn{2}{|c|}{0.51} & & \\
\hline \multirow{3}{*}{ Int. rotation } & Taping & 0.68 & 0.37 & 0.07 & 2.98 \\
\hline & Control & 0.45 & 0.14 & 0.03 & \\
\hline & Diff & & 0.23 & & \\
\hline \multirow{3}{*}{ Ext. rotation } & Taping & 1.39 & 0.48 & 0.09 & 5.93 \\
\hline & Control & 0.73 & 0.31 & 0.06 & \\
\hline & Diff & & 0.64 & & \\
\hline
\end{tabular}

ment. Muscle and collagenous tissue are both very adaptable, and studies have shown that low-load, long duration stretching is more effective than short-term, vigorous stretching $[17,18]$.Taping may be one way to achieve this low-load, prolonged-duration stretching.

Secondly, therapeutic exercise has previously been determined to have long-term benefits for patients with SIS $[19,20]$. Based on the significant improvement in strength in the scapular taping group, the application of scapular taping appeared to optimize conditions for performing the stretching and strengthening exercises. These optimum conditions may be due to the significant pain reduction and increased pain-free ROM immediately following the application of scapular taping procedures.

Thirdly, adding to it the conventional exercise therapy might reduce pain by stimulating joint mechanoreceptor activity, which, in turn, is thought to block aberrant afferent pain signals and reduce the awareness of pain [21]. It has also been hypothesized that stretching exercises mechanically stretches shortened collagenous tissue and improves interstitial fluid content resulting in restoration of movement [22]. Similar to the relationship noted between pain and muscle strength in this study, increased pain was associated with decreased muscle strength at baseline, and the changes in pain over 6 weeks correlated with changes in muscle strength. This negative association between pain and muscle strength detected in this investigation has some precedence in previous studies which have suggested that pain influences muscle activation as well as maximum voluntary muscle strength [23].

There have been few studies of therapeutic outcomes and expected durations of treatment regarding conservative management of SIS. Several authors imply that if the patient with shoulder impingement was not better after 3 months of conservative treatment, surgery was indicated $[24,25]$. The patients in this study were treated for 6 weeks for a total of 18 visits. It is felt that through the use of scapular taping, the treatment was of shorter duration than it would have been without the taping technique.

\section{CONCLUSION}

Scapular taping may be a useful adjunct for promoting proper scapular position and can be used in conjunction with other interventions, like specifically selected exercises, patient education and about modification of performing overhead activities. A thorough examination of the scapula's position at rest and during movements is most important before using taping as a treatment technique.

\section{REFERENCES}

[1] Van der Windt, D.A., Koes, B.W., Boeke, A.J., Devillé, W., De Jong, B.A. and Bouter, L.M. (1996) Shoulder disorders in general practice: Prognostic indicators of outcome. British Journal of General Practice, 46, 519-523.

[2] Gorski, J.M. and Schwartz, L.H. (2003) Shoulder impingement presenting as neck pain. Journal of Bone and Joint Surgery, 85, 635-638.

[3] Borstad, J.D. (2006) Resting position variables at the shoulder: Evidence to support posture impairment association. Physical Therapy, 86, 549-567.

[4] Lewis, J.S., Green, A.S. and Dekel, S. (2001) The etiology of subacromial impingement syndrome. Physiotherapy, 87, 458-469. doi:10.1016/S0031-9406(05)60693-1

[5] Lewis, J.S., Wright, C. and Green, A. (2005) Subacromial impingement syndrome: The effect of changing posture on shoulder range of movement. Journal of Orthopaedic and Sports Physical Therapy. 35, 72-87.

[6] Chard, M.D., Sattelle, L.M. and Hazleman, B.L. (1988) The long term outcome of rotator cuff tendinitis-review study. British Journal of Rheumatology, 27, 385-389. doi:10.1093/rheumatology/27.5.385

[7] Park, H.B., Yokota, A., Gill, H.S., Rassi, G.E. and McFarland, E.G. (2005) Diagnostic accuracy of clinical tests for the different degrees of subacromial impingement syndrome. Journal of Bone and Joint Surgery, 87, 1446-1455. doi:10.2106/JBJS.D.02335 
[8] Lewis, J.S., Green, A.S. and Wright, C. (2005) Subacromial impingement syndrome: The role of posture and muscle imbalance. Journal of Shoulder and Elbow surgery, 14, 385392. doi:10.1016/j.jse.2004.08.007

[9] Darlow, B. (2006) Neuromuscular retraining for multidirectional instability of the shoulder-A case study. New Zealand Journal of Physiotherapy, 34, 60-65.

[10] Johnston, T. (1937) The movements of the shoulder joint: A plea for the "plane of scapula" as a reference for movements occurring at the humeroscapular joint. British Journal of Surgery, 25, 252-260. doi:10.1002/bjs.1800259803

[11] Warner, J., Micheli, L., Kennedy, A.L. and Kennedy, R. (1992) Scapulothoracic motion in normal shoulders and shoulders with glenohumeral instability and impingement syndrome: A study using Moire topography analysis. Clinical Orthopaedics and Related Research, 285, 191-199.

[12] Greenfield, B., Catlin, P., Coats, P., Green, E., McDonald, J. and North, C. (1995) Posture in patients with shoulder overuse injuries and healthy individuals. Journal of Orthopedic and Sports Physical Therapy, 21, 287-295.

[13] Heald, S.L., Riddle, D.L. and Lamb, R.L. (1997) The shoulder pain and disability index: The construct validity and responsiveness of a region-specific disability measure. Physical Therapy, 77, 1079-1089.

[14] MacDermid, J.C., Solomon, P. and Prkachin, K. (2006) The shoulder pain and disability index demonstrates factor, construct and longitudinal validity. BMC Musculoskeletal Disorders, 7, 7-18.

[15] Williams, J.W., Holleman, D.R. Jr. and Simel, D.L. (1995) Measuring shoulder function with the shoulder pain and disability index. Journal of Rheumatology, 22, 727-732.

[16] Hayes, K., Walton, J.R., Szomor, Z.L. and Murrell, G.C. (2002) Reliability of 3 methods for assessing shoulder strength. Journal of Shoulder and Elbow Surgery, 11, 33-39. doi:10.1067/mse.2002.119852

[17] Kottke, F.J., Pauley, D.L. and Ptak, R.A. (1966) The rationale for prolonged stretching for correction of shortening of connective tissue. Archives of Physical Medicine and Rehabilitation, 47, 345-352.

[18] Williams, P.E. and Goldspink, G. (1978) Changes in sarcomere length and physiological properties in immobilized muscle. Journal of Anatomy, 127, 459-468.

[19] Bang, M.D. and Deyle, G.D. (2000) Comparison of supervised with and without manual physical therapy for patients with shoulder impingement syndrome. Journal of Orthopaedic and Sports Physical Therapy, 30, 126-137.

[20] Dickens, V.A., Williams, J.L. and Bhamra, M.S. (2005) Role of physiotherapy in the treatment of subacromial impingement syndrome: A prospective study. Physiotherapy, 91, 159-164. doi:10.1016/j.physio.2004.10.008

[21] Sauers, E.L. (2005) Effectiveness of rehabilitation for patients with subacromial impingement syndrome. Journal of Athletic Training, 49, 221-223

[22] Dorrestijn, O., Stevens1, M., Diercks1, R.L., Van der Meer, K. and Winters, J.C. (2007) A new interdisciplinary treatment strategy versus usual medical care for the treatment of subacromial impingement syndrome: A randomized controlled trial. BMC Musculoskeletal Disorders, 8, 15.

[23] Hawkins, R.F. and Kennedy, J.C. (1980) Impingement syndromes in athletes. American Journal of Sports Medicine, 8, 151-158. doi:10.1177/036354658000800302

[24] Penny, J.N. and Welsh, M.B. (1981) Shoulder impingement syndromes in athletes and their surgical management. American Journal of Sports Medicine, 9, 11-15. doi:10.1177/036354658100900102

[25] Jobe, F.W. and Bradley, J.P. (1989) The diagnosis and nonoperative treatment of shoulder injuries in athletes. Clinics in Sports Medicine, 8, 419-438. 